\title{
Stability and modulation properties of a semiconductor laser with weak optical feedback from a distant reflector
}

\author{
C Masoller $\ddagger$ and N B Abraham $\ddagger$ \\ $\dagger$ Instituto de Fisica, Facultad de Ciencias, Tristan Narvaja 1674, Montevideo, Uruguay \\ $\ddagger$ Department of Physics, Bryn Mawr College, 101 N Merion Ave, Bryn Mawr, PA, 19010-2899, \\ USA
}

Received 4 November 1997, in final form 12 February 1998

\begin{abstract}
We analyse the stability of the steady-state solutions of the compound system formed by a single-longitudinal-mode semiconductor laser and an external reflector. Of the large number of these external cavity modes (ECMs) created in pairs of modes and antimodes by moderate feedback, the antimodes are always unstable, while the modes may be stable or unstable when created. The ECMs that have a large positive frequency shift with respect to the emission frequency of the solitary laser are unstable when created. In contrast, the ECMs that have a large, negative frequency shift are stable on creation and remain stable over a relatively large feedback range. For sufficiently large feedback an ECM that is created stable gives way to a time-dependent solution (limit cycle or torus) that is localized in phase space around the ECM; several time-dependent attractors may coexist. Approximating the dynamical equations, taking into account terms up to second order, we obtain relations for the amplitudes of the oscillations of the laser variables for these attractors. The external cavity length and the injection current are key parameters which determine these amplitudes. Our approximations are in good agreement with numerical simulations, and hold even far from the bifurcation points where these attractors originate.
\end{abstract}

\section{Introduction}

Optical feedback from a short external cavity is commonly used to improve the spectral properties of a single-longitudinal-mode semiconductor laser (to narrow the linewidth, to reduce the intensity noise and to improve the frequency stability) [1]. In contrast, even weak feedback (of the order of $\sim 10^{-6}-10^{-1} \%$ of the emitted intensity) from a long external cavity leads to the excitation of many external cavity modes (ECMs). The ECMs are not modes of the empty cavity (which form an infinite set), but a finite set of self-consistent steady-state solutions of the compound system, formed by the laser and the external reflector. We chose a model for the laser, which in the absence of feedback describes operation in a single (longitudinal) mode, but this might be said to become multimode in the presence of feedback, in the sense that it might operate on several external cavity modes. The frequency of the solitary laser mode is slightly modified by the feedback, which in addition introduces new modes (the ECMs), which are located in the frequency domain around the solitary laser frequency. For very low feedback levels many of the ECMs are stable, and competition among the steady-state solutions can occur. For larger feedback the mode competition is suppressed and the laser operates in a single ECM, usually the ECM with the lowest linewidth. Moderate feedback induces a periodic modulation of the laser output. Even larger feedback induces a chaotic state, with a broadband emission, termed coherence 
collapse $[2,3]$. The sensitivity of these systems to giving way to time-dependent solutions may affect how lasers can be used in optical communications systems, where laser diodes coupled to optical fibres are routinely employed and for which external reflections cannot be completely avoided.

For weak and moderately strong feedback levels many features of the dynamics of a semiconductor laser can be described by the Lang and Kobayashi model [4], which consists of two rate equations, one for the slowly varying amplitude of the intracavity complex electric field of a single longitudinal mode of the solitary laser and one for the carrier density in the semiconductor medium. The external cavity is described by two parameters: the strength of the feedback, $\gamma$, and the delay time, $\tau$, which is proportional to the external cavity length. The equation for the evolution of the field amplitude contains a time-delayed term to describe the field reflected form the external mirror (multiple reflections are neglected).

The external cavity modes are steady-state solutions of the Lang and Kobayashi equations and their number increases with either $\gamma$ or $\tau$. The steady-state solutions appear in pairs; in each pair one is called a mode (associated with constructive interference of the field inside the laser cavity and the field returning form the external mirror) and the other is called an antimode (associated with destructive interference). The stability of a particular steady-state solution is determined by the real part of the roots of a complicated transcendental equation for the complex growth rates of small perturbations from that solution (see, for example, equation (41) of [5], where the effect of nonlinear gain suppression was not included, or equation (2.15) of [6], where nonlinear gain suppression was taken into account). Two distinct types of instabilities have been identified: saddlenode instabilities and Hopf instabilities. In a saddle-node instability there is one positive root, which means that the steady-state solution is an unstable fixed point (an 'antimode'). In a Hopf instability there are two complex-conjugate roots with positive real parts, and the instability corresponds to the growth of periodic modulations about the steady-state solution when it is perturbed (this is typically associated with the destabilization of the relaxation oscillations around the ECM, as the feedback is increased).

Several previous investigations have explored the asymptotic behaviour when the relaxation oscillations are undamped. Using linear stability analysis Ritter and Haug determined the location of the Hopf bifurcation points [6]. Similar results were obtained by Erneux et al [7] using asymptotic methods, in an approximation based on the small value of the ratio of the photon and carrier lifetimes, and the assumption that the laser is biased well above threshold. The criterion for a Hopf bifurcation for low values of the pump current was examined in $[8,9]$ using singular perturbation methods. Levine et al [10] proved that there always exists a feedback phase condition where a special ECM (one which they call the maximum gain mode) is stable for arbitrarily large feedback strengths. Increasing feedback levels destabilize relaxation oscillations around this mode, but before this occurs, a new stable mode has appeared, which is the 'new' maximum gain mode.

By numerical simulation of the Lang and Kobayashi equations, we recently studied the coexisting time-dependent attractors that develop locally in phase space from the ECMs as the feedback strength is increased, for a laser biased well above threshold and subjected to distant external reflection [11]. These 'ECM attractors' can be classified into distinct categories, depending on the relation of the optical frequency, $\omega_{i}$, of the ECM from which the attractor originates to the solitary laser optical frequency, $\omega_{0}$. When the laser operates on an ECM with $\omega_{i} \ll \omega_{0}$, the laser intensity is constant over a relatively large feedback range (the maximum gain mode of [10] belongs to this category). In contrast, in our numerical simulations we did not find any attracting solution that develops from ECMs with $\omega_{i} \gg \omega_{0}$. 
When the laser operates on one of the ECMs with a frequency not much larger than $\omega_{0}$, as the feedback increases a periodic or quasiperiodic modulation of the laser output appears (i.e. the ECM attractor is a limit cycle or a torus). The so-called minimum linewidth mode, which is the ECM in the deepest potential well of the 'thermodynamic' potential model $[12,13]$, belongs to this category.

These previous studies raised the question: is it possible to observe the laser operating on ECMs with $\omega_{i} \gg \omega_{0}$ ? We have divided our analysis into two parts. In the first part we study the stability of the external cavity modes, and in the second part, we analyse the characteristics of the modulation induced by the external feedback.

We show analytically that these modes do not become unstable as the feedback increases, but rather they are already unstable when they first appear. For increasing feedback the pairs of steady-state solutions which have $\omega_{i} \gg \omega_{0}$ are both created unstable, one an unstable mode and the other an unstable antimode. For these pairs the Hopf bifurcation occurs in the antimodes. This bifurcation does not create a stable periodic behaviour because the fixed point that undergoes the bifurcation has a positive real eigenvalue. However, experimentally Mørk et al [14] found what appeared to be a similar type of subcritical Hopf bifurcation that created stable periodic behaviour: at a certain feedback level an ECM becomes unstable and a stable limit cycle appears, while at a higher feedback level, when another pair of complexconjugate eigenvalues acquire positive real parts, another stable limit cycle appears, which is created from the fixed point (now unstable). The observation of bistability between limit cycles evolved from the same ECM was explained theoretically in [15].

Ritter and Haug [16] showed that, near the Hopf bifurcation where the oscillations originate, the relation between the amplitude of the limit cycle and the excess of the feedback parameter above the bifurcation point is governed by the typical square-root law of supercritical Hopf bifurcations. More recently, Alsing et al [17] derived a third-order delay differential equation for the phase of the laser field (using asymptotic methods based on the small ratio of the photon and carrier lifetimes, and on the relatively large value of the linewidth enhancement factor). For this equation they proved that in a feedback region in which only one ECM exists, there are coexisting periodic solutions that surround the ECM, with amplitudes that are proportional to the roots of the Bessel function $J_{1}(x)$. The limit cycle associated with the first root arises from a Hopf bifurcation of the ECM, while the others arise from saddle-node bifurcations. The authors interpret the periodic solutions surrounding the external cavity mode as a new set of external cavity modes, with a more complicated time dependence.

For a laser biased well above threshold and subjected to distant external reflection, we found in [11] that when it operates in an ECM with a frequency $\omega_{i} \approx \bar{\omega}$ (where $\bar{\omega}$ is slightly larger than $\omega_{0}$ ), moderate feedback destabilizes the ECM, inducing a periodic modulation over a wide range of feedback. The ECMs with frequency slightly larger than $\omega_{0}$, which are the first to become unstable as the feedback increases, give rise to the most stable ECM attractors (in the sense that the periodic solutions are the last to become destabilized by the feedback). When the laser operates on ECMs with $\omega_{i}>\bar{\omega}$ or $\omega_{i}<\bar{\omega}$ the periodic modulation occurs in an extremely narrow feedback range, followed almost immediately by a second Hopf bifurcation that gives rise to quasiperiodic modulation. The quasiperiodic modulation has two clearly distinct stages: one of large, almost periodic oscillations (that resemble the periodic modulation), and another of small oscillations. In the first stage (which we call the relaxation-oscillation stage) the amplitudes of the modulations of the laser variables are similar for each of the different ECM attractors, and the laser has a mean optical frequency, $\langle\omega\rangle$, that is approximately equal to $\bar{\omega}$. In the second stage the evolution occurs close to the unstable (steady-state) ECM, and therefore $\langle\omega\rangle \approx \omega_{i}$. 
In this paper we explain these numerical results by deriving analytical relations for the amplitudes of the modulations of the laser variable in the relaxation-oscillation stage, and an equation for $\langle\omega\rangle$ in this stage. Our results agree with those of [16] in the domain of its validity, but also hold for feedback levels well above the onset of periodic oscillations.

The remainder of this paper is organized as follows. Section 2 contains a brief description of the model and the stability analysis of the ECMs, modes and antimodes. In section 2 we approximate the Lang and Kobayashi equations, taking into account terms up to second order, and we derive relations for the amplitudes of the modulations of the laser variables in the ECM attractors. We compare our results with numerical simulations of the original rate equations. Section 4 provides a summary and conclusions.

\section{Stability of the external cavity modes}

Writing the slowly varying amplitude of the intracavity electric field as $E(t) \exp \left[\mathrm{i}\left(\omega_{0} t+\right.\right.$ $\phi(t))$ ], where $E$ and $\phi$ are real and $\omega_{0}$ is the laser frequency without feedback, the Lang and Kobayashi rate equations are [4]

$$
\begin{aligned}
\frac{\mathrm{d} E(t)}{\mathrm{d} t} & =\frac{1}{2}\left[G\left(N, E^{2}\right)-\frac{1}{\tau_{\mathrm{p}}}\right] E(t)+\gamma E(t-\tau) \cos \left[\omega_{0} \tau+\Delta \phi(t)\right] \\
\frac{\mathrm{d} \phi(t)}{\mathrm{d} t} & =\frac{\alpha}{2}\left[G\left(N, E^{2}\right)-\frac{1}{\tau_{\mathrm{p}}}\right]-\gamma \frac{E(t-\tau)}{E(t)} \sin \left[\omega_{0} \tau+\Delta \phi(t)\right] \\
\frac{\mathrm{d} N(t)}{\mathrm{d} t} & =J-\frac{N(t)}{\tau_{\mathrm{s}}}-G\left(N, E^{2}\right) E(t)^{2} .
\end{aligned}
$$

The modulus of the electric field $E(t)$ is normalized such that $V_{\mathrm{c}} E(t)^{2}$ is the total photon number in the laser waveguide (where $V_{\mathrm{c}}$ is the volume of the active region). $N(t)$ is the carrier population. $\Delta \phi(t)=\phi(t)-\phi(t-\tau)$ is the phase change after one round trip in the external cavity. The other parameters are $\tau_{\mathrm{s}}$, the carrier lifetime; $\tau_{\mathrm{p}}$, the photon lifetime and $G$, the gain per unit time $G=G_{N}\left(N-N_{0}\right)\left(1-\varepsilon E^{2}\right)$, where $G_{N}$ is the modal gain coefficient, $N_{0}$ is the carrier density at transparency and $\varepsilon$ the nonlinear gain coefficient. $\alpha$ is the linewidth enhancement factor and $J$ is the current density in carriers per unit volume and unit time.

The steady-state solutions are obtained by substituting $E(t)=E_{\mathrm{s}}, \phi(t)=\left(\omega_{i}-\omega_{0}\right) t$ and $N(t)=N_{\mathrm{s}}$ in equations (1)-(3). Their frequencies $\omega_{i}$ are the solutions of

$$
f(\omega \tau) \equiv \omega \tau-\omega_{0} \tau+\gamma \tau \sqrt{1+\alpha^{2}} \sin (\omega \tau+\arctan \alpha)=0 .
$$

The carrier density and field amplitude of a given solution are found by solving

$$
G_{\mathrm{s}, i}=G_{N}\left(N_{\mathrm{s}, i}-N_{0}\right)\left(1-\varepsilon E_{\mathrm{s}, i}^{2}\right)=1 / \tau_{\mathrm{p}}-2 \gamma \cos \left(\omega_{i} \tau\right)
$$

and

$$
J-N_{\mathrm{s}, i} / \tau_{\mathrm{s}}-G_{\mathrm{s}, i} E_{\mathrm{s}, i}^{2}=0 .
$$

Equation (4) admits several solutions and their number increases as $C=\gamma \tau \sqrt{1+\alpha^{2}}$ increases. Figure 1 illustrates a typical situation with a graphical solution of equation (4) for $\gamma=1.5 \mathrm{GHz}, \tau=5 \mathrm{~ns}, \alpha=4.4$ and $\omega_{0}=6 \mathrm{rad}$. There are 21 steady-state solutions. The stability of each stationary solution can be determined by a linear stability analysis of the rate equations in the vicinity of the solution. The solutions with frequencies that satisfy

$$
1+\gamma \tau \sqrt{1+\alpha^{2}} \cos \left(\omega_{i} \tau+\arctan \alpha\right)<0
$$




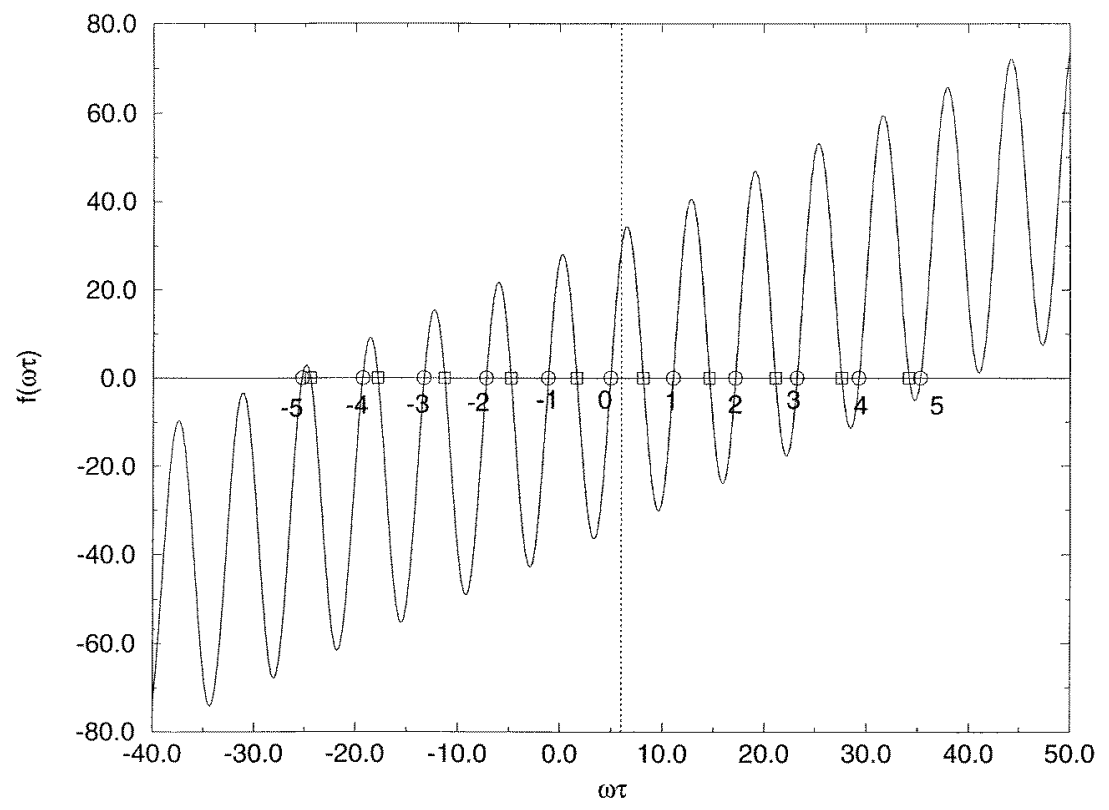

Figure 1. Graphical solution of equation (4) for $\gamma=1.5 \mathrm{GHz}, \tau=5 \mathrm{~ns}, \alpha=4.4$ and $\omega_{0} \tau=6 \mathrm{rad}$. The circles indicate the modes, the squares the antimodes and the dotted line the value of $\omega_{0} \tau$.

have a real, positive eigenvalue and are therefore unstable saddle ('antimodes'). The other solutions are termed 'modes'. The antimodes are the solutions for which the slope of $f(\omega \tau)$ is negative. When a pair of solutions appears, one is always an antimode.

The feedback level at which a saddle-node bifurcation occurs and a pair of solutions of frequency $\omega_{i}^{\mathrm{sn}}$ appears is [11]

$$
\gamma_{i}^{\mathrm{sn}}=\frac{-1}{\tau \sqrt{1+\alpha^{2}} \cos \left(\omega_{i}^{\mathrm{sn}} \tau+\arctan \alpha\right)}
$$

where $\omega_{i}^{\mathrm{sn}}$ is a solution of

$$
\omega_{i} \tau-\omega_{0} \tau=\tan \left(\omega_{i} \tau+\arctan \alpha\right) .
$$

If $\omega_{\mathrm{R}} \tau=(2 m+1) \pi$ (where $\omega_{\mathrm{R}}=\sqrt{G_{N}\left(J-J_{\mathrm{th}}\right)}$ is the relaxation-oscillation angular frequency of the solitary laser), a good approximation of the feedback rate at which a Hopf bifurcation occurs in the ECM with frequency $\omega_{i}$ is $[6,10]$

$$
\gamma_{i}^{\mathrm{H}}=\frac{-\lambda_{\mathrm{R}}}{\sqrt{1+\alpha^{2}} \cos \left(\omega_{i} \tau+\arctan \alpha\right)}
$$

where $\lambda_{\mathrm{R}}=\left[1 / \tau_{\mathrm{s}}+\left(\tau_{\mathrm{p}}+\varepsilon / G_{N}\right) \omega_{\mathrm{R}}^{2}\right] / 2$ is the damping rate of the solitary laser. (For this feedback level, the ECM with frequency $\omega_{i}$ has a pair of complex-conjugate eigenvalues with zero real part, $s= \pm \mathrm{i} \Omega$, with $\Omega \approx \omega_{\mathrm{R}}$.)

Figure 2(a) shows, for the same parameter values as figure 1 and $\lambda_{\mathrm{R}}=3.8 \mathrm{GHz}$, the feedback levels at which saddle-node and Hopf bifurcations occur. For $\gamma=1.5 \mathrm{GHz}$ (broken curve) we have five pairs of solutions with mode number $i<0$, five with $i>0$ and the $i=0$ mode. For the ECMs with mode number $i=-3,-4$ and $-5, \gamma^{\mathrm{H}}$ is greater than $1.5 \mathrm{GHz}$, thus these modes are stable. The most unstable solution has mode number $i=2$ (the one which has lowest $\gamma^{\mathrm{H}}$ ). 

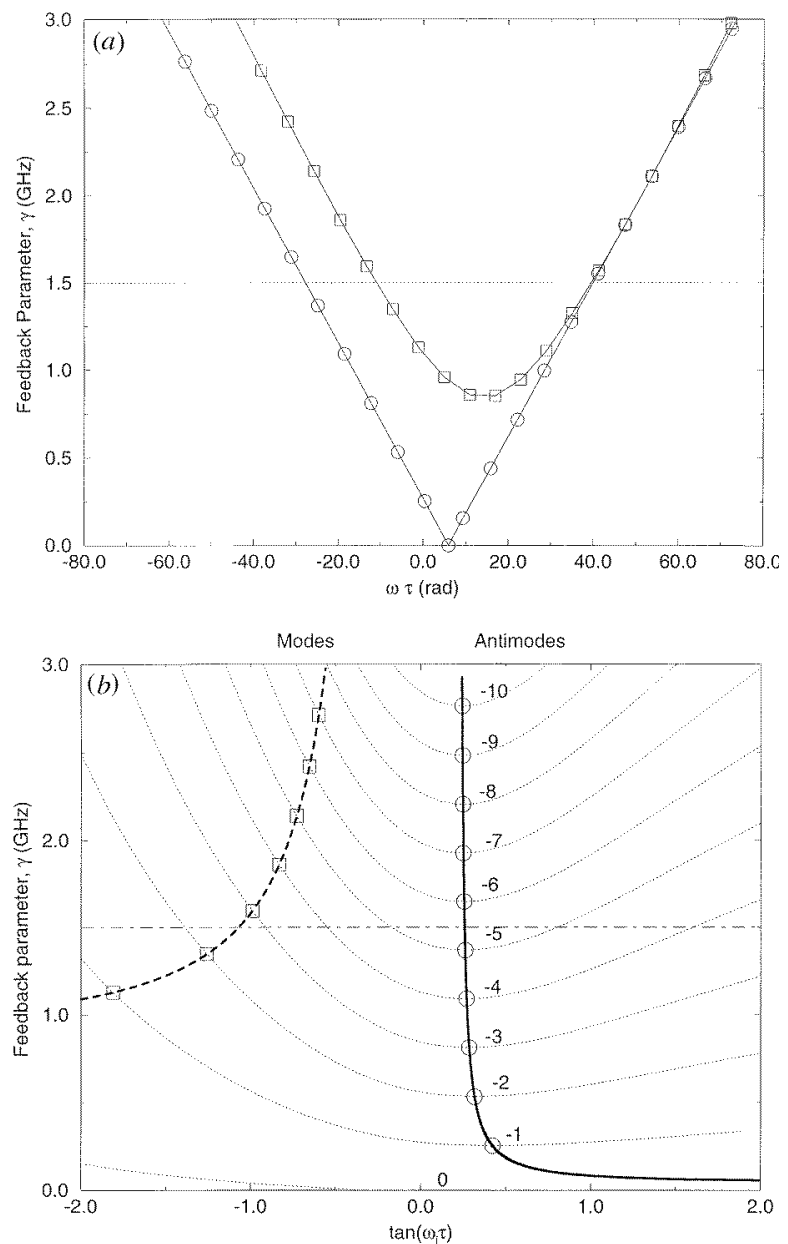

Figure 2. (a) Saddle-node and Hopf curves in the $(\omega \tau, \gamma)$ plane. The circles indicate where pairs of ECMs appear, and the squares, where the ECMs undergo Hopf bifurcations. The ECMs are labelled in accordance with figure 1. The parameters are $\tau=5 \mathrm{~ns}, \alpha=4.4$, $\omega_{0} \tau=6 \mathrm{rad}$ and $\lambda_{\mathrm{R}}=3.8 \mathrm{GHz}$. (b) Variation of the external cavity modes (modes and antimodes) with negative mode number in the $\left(\tan \left(\omega_{i} \tau\right), \gamma\right)$ plane. The saddle-node curve (full) was calculated from equation (8), and the Hopf curve (broken) from equation (10). The evolution of the ECMs in this plane (dotted) was calculated from equation (4). With increasing feedback, the modes and antimodes separate in phase space. The modes move to the left and the antimodes to the right. The circles indicate where a pair of ECMs appear, and the squares indicate where a Hopf bifurcation occurs. (c) Evolution of the ECMs (modes and antimodes) with positive mode number. The modes move to the right and the antimodes to the left with increasing $\gamma$.

The bifurcation diagram is similar to that of a laser diode with monochromatic external optical injection $[18,19]$. In the (input field frequency, input field amplitude) plane, the curve above which relaxation oscillations become undamped 'touches' the line below which locking cannot be achieved in the positive frequency region, while it is above and almost parallel to this line in the negative frequency region (see, for example, figures 4 and 5 of [18], or figure 1 of [19]). 


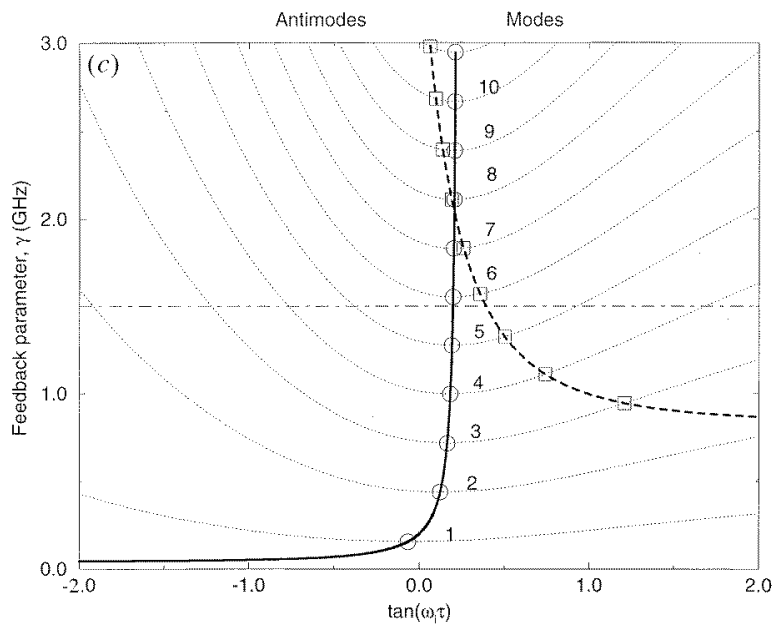

Figure 2. Continued.

To display the stability of the steady-state solutions in more detail, figure $2(b)$ shows the evolution in the $\left(\tan \left(\omega_{i} \tau\right), \gamma\right)$ plane of the solutions with $i<0$, and figure 2(c) shows the evolution of the solutions with $i>0$. On the saddle-node curve (full curve) pairs of solutions appear. As $\gamma$ grows the modes and antimodes separate in phase space. The modes with $i<0$ move to the left, and the antimodes move to the right (figure 2(b)), while the modes with $i>0$ move to the right and the antimodes move to the left (figure 2(c)). (If $\gamma$ increases and $\omega_{0} \tau$ is fixed, the amplitude of the sine term in (4) grows, and in figure 1 the modes with $i>0$ move to the right, decreasing their frequency, the modes with $i<0$ move to the left, increasing their frequency, while the frequency of the antimodes decreases.)

When a solution crosses the Hopf boundary (broken curve), two complex-conjugate eigenvalues of the linearized system change the sign of their real parts. If the ECM is a stable mode, relaxation oscillations around this mode become undamped and self-sustained oscillations (of frequency approximately equal to $\omega_{\mathrm{R}}$ ) appear; if the ECM is unstable, the mode remains unstable but the dynamical transient changes, giving evidence of the Hopf bifurcation which adds complex-conjugate eigenvalues with positive real parts to the eigenvalues with positive real parts.

We first consider the stability of the ECMs with $\omega_{i}<\omega_{0}$. In figure $2(b)$, for $\gamma=1.5 \mathrm{GHz}$ (this value corresponds to figure 1 and is indicated by a chain curve) the ECMs with $i=-3,-4$ and -5 are below the Hopf curve and therefore have not become unstable yet; the other ECMs have already crossed the curve and lost stability.

The modes with large negative mode number (which appear for moderately strong feedback) cross the Hopf curve at high feedback levels. This agrees with the stability analysis of Levine et al [10]. (The maximum gain mode is the ECM with maximum negative frequency shift with respect to $\omega_{0}$; see figure 1 of [10].) However, numerical simulations reveal that these ECMs have narrow basins of attraction [11].

Next we analyse the stability of the ECMs with $\omega_{i}>\omega_{0}$. As shown in figure 2(c), the ECMs with large positive mode number (which appear for moderately strong feedback) are created unstable, because when they first appear they are above the Hopf curve. For increasing $\gamma$ the modes move to the right and the antimodes to the left. Therefore, the antimodes cross the Hopf curve and undergo a Hopf bifurcation. We have found these bifurcations numerically, by integrating equations (1)-(3). A fourth-order Runge-Kutta 
Table 1. Parameter values used in the numerical simulations.

\begin{tabular}{ll}
\hline$\alpha$ & 4.4 \\
$\omega_{0} \tau$ & $6 \mathrm{rad}$ \\
$\tau_{\mathrm{p}}$ & $0.0014 \mathrm{~ns}$ \\
$\tau_{\mathrm{s}}$ & $1 \mathrm{~ns}$ \\
$J / J_{\mathrm{th}}$ & 2 \\
$\omega_{\mathrm{R}}$ & $42 \mathrm{GHz}$ \\
$G_{N}$ & $839 \times 10^{-24} \mathrm{~m}^{3} \mathrm{~ns}^{-1}$ \\
$\Delta J$ & 1.23 \\
$\xi$ & 1.40 \\
$\varepsilon$ & $2 \times 10^{-24} \mathrm{~m}^{3}$ \\
$\varepsilon^{\prime}$ & 0.0048 \\
$\varepsilon^{\prime} \Delta J$ & 0.0058 \\
$\varepsilon^{\prime \prime}$ & 0.05 \\
\hline
\end{tabular}
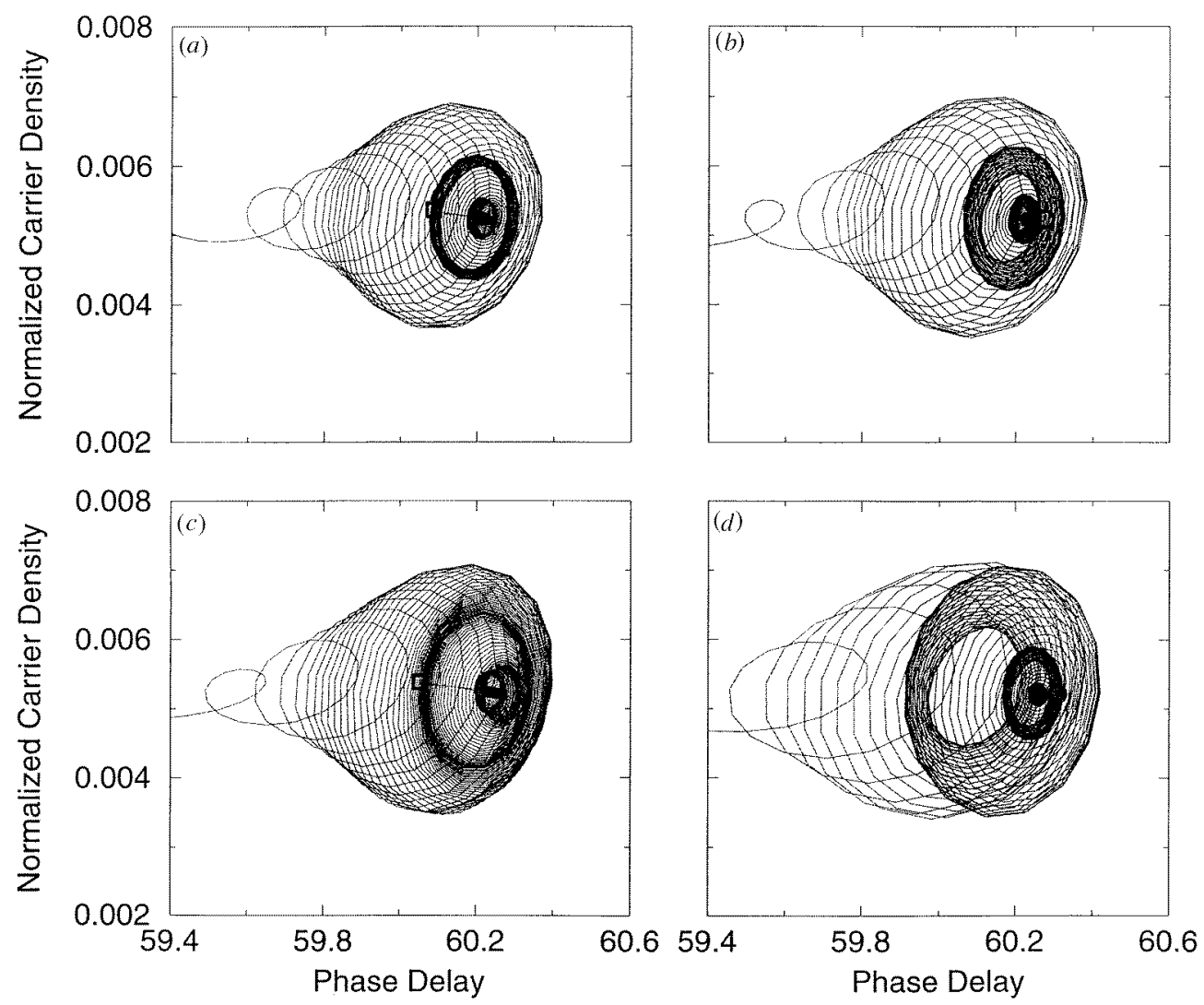

Figure 3. Numerical simulation of equations (1)-(3). (a) Initial conditions in antimode $i=10$ (indicated with a square), $\gamma=2.68 \mathrm{GHz}$. (b) Initial conditions in mode $i=10$ (indicated with a circle), $\gamma=2.68 \mathrm{GHz}$. (c) Initial conditions in antimode $i=10, \gamma=2.69 \mathrm{GHz}$. (d) Initial conditions in mode $i=10, \gamma=2.69 \mathrm{GHz}$.

method was used (with an integration step $\Delta t=0.001 \mathrm{ns)}$ with the laser parameters of table 1, which are the same as in [11]. We have taken the external cavity parameters to be $\tau=5 \mathrm{~ns}$ and $\gamma$ slightly below and above the Hopf bifurcation point. As an example, 
figure 3 shows the results for initial conditions in the mode and antimode with mode number $i=10\left(\gamma_{10}^{\mathrm{H}}=2.684 \mathrm{GHz}\right)$. Both the antimode and the mode are already unstable below $\gamma_{10}^{\mathrm{H}}$, and after a transient, the trajectory leaves the vicinity of these modes (figures $3(a)$ and $(b)$ ). Above $\gamma_{10}^{\mathrm{H}}$ they are also unstable, but the transient is different, suggesting the existence of a new unstable quasiperiodic solution (figures $3(c)$ and $(d)$ ).

\section{Relaxation-oscillation phenomena}

Next, we study the characteristics of the modulation induced by moderate feedback levels, when the laser operates on ECM attractors with frequencies near $\omega_{0}$. As an example, figure 4 shows four coexisting attractors of equations (1)-(3). The external cavity parameters are $\gamma=1.2 \mathrm{GHz}$ and $\tau=10 \mathrm{~ns}$. The variables $e$ and $n$ are defined below, in equation (20). From ECM modes $i=-1,1$ and 6, quasiperiodic tori have developed (figures 4(a), $(b)$ and $(d)$ ); from mode $i=3$ a limit cycle has developed (figure 4(c)).

The feedback levels above which relaxation oscillations around these modes become undamped are $\gamma_{-1}^{\mathrm{H}}=1.017, \gamma_{1}^{\mathrm{H}}=0.885, \gamma_{3}^{\mathrm{H}}=0.844$ and $\gamma_{6}^{\mathrm{H}}=0.973 \mathrm{GHz}$. Therefore, the feedback level $\gamma=1.2 \mathrm{GHz}$ is $18 \%, 35 \%, 42 \%$ and $23 \%$ above $\gamma_{-1}^{\mathrm{H}}, \gamma_{1}^{\mathrm{H}}, \gamma_{3}^{\mathrm{H}}$ and $\gamma_{6}^{\mathrm{H}}$, respectively. Note, however, that in the four ECM attractors the relaxation-oscillation stage is similar, almost an ellipse in the phase space, and that the amplitudes of the oscillations are almost equal.
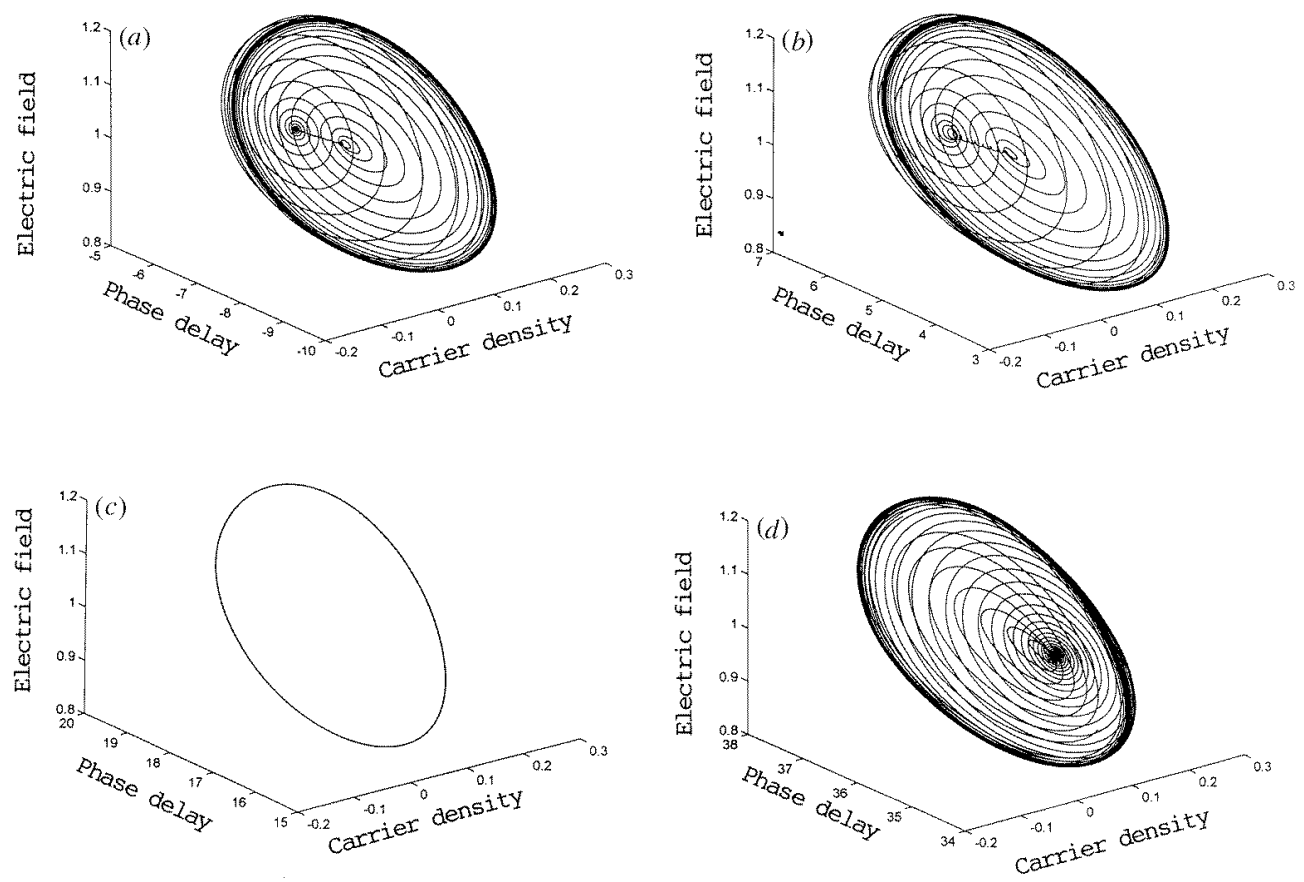

Figure 4. Coexisting ECM attractors, for $\gamma=1.2 \mathrm{GHz}, \tau=10 \mathrm{~ns}$. The $x$-axis represents the carrier density $n(t)$, the $y$-axis the phase delay $\Delta \phi(t)$, and the $z$-axis the electric field $1+e(t)$. (a), (b) Quasiperiodic tori developed from ECMs $i=-1$ and $i=1$; (c) limit cycle developed from ECM $i=3 ;(d)$ quasiperiodic torus developed from ECM $i=6$. 
Our goal is to obtain relations for the amplitudes of the oscillations and mean values of the laser variables in the relaxation-oscillation stage. We rescale the variables by introducing

$$
E=\sqrt{\frac{1}{2} \tau_{\mathrm{s}} G_{N}} E \quad \Delta N=\frac{1}{2} \tau_{\mathrm{p}} G_{N}\left(N-N_{\text {th }}\right)
$$

where $N_{\text {th }}$ is the threshold current density. The gain function can be written as

$$
G\left(\Delta N, E^{2}\right)=\frac{1}{\tau_{\mathrm{p}}}+\frac{2 \Delta N}{\tau_{\mathrm{p}}}-\varepsilon^{\prime}\left(\frac{1}{\tau_{\mathrm{p}}}+\frac{2 \Delta N}{\tau_{\mathrm{p}}}\right) E^{2}
$$

where $\varepsilon^{\prime}=2 \varepsilon / \tau_{\mathrm{s}} G_{N}$. Equations (1)-(3) can be re-written as

$$
\begin{aligned}
& \frac{\mathrm{d} E}{\mathrm{~d} t}=\frac{\Delta N E}{\tau_{\mathrm{p}}}-\frac{\varepsilon^{\prime}}{2 \tau_{\mathrm{p}}}(1+2 \Delta N) E^{3}+\gamma E(t-\tau) \cos \left(\omega_{0} \tau+\Delta \phi\right) \\
& \frac{\mathrm{d} \phi}{\mathrm{d} t}=\frac{\alpha \Delta N}{\tau_{\mathrm{p}}}-\frac{\alpha \varepsilon^{\prime}}{2 \tau_{\mathrm{p}}}(1+2 \Delta N) E^{2}-\gamma \frac{E(t-\tau)}{E(t)} \sin \left(\omega_{0} \tau+\Delta \phi\right) \\
& \tau_{\mathrm{s}} \frac{\mathrm{d} \Delta N}{\mathrm{~d} t}=\Delta J-\Delta N-(1+2 \Delta N) E^{2}+\varepsilon^{\prime}(1+2 \Delta N) E^{4}
\end{aligned}
$$

where $\Delta J=\tau_{\mathrm{p}} \tau_{\mathrm{s}} G_{N}\left(J-J_{\mathrm{th}}\right) / 2=\tau_{\mathrm{p}} \tau_{\mathrm{s}} \omega_{\mathrm{R}}^{2} / 2$. Making another change of variables

$$
E=\sqrt{\Delta J}(1+e) \quad \phi=\varphi-\omega_{0} t \quad n=\frac{\Delta N}{\omega_{\mathrm{R}} \tau_{\mathrm{p}}}
$$

and assuming that $\Delta J \neq 0$ leads to

$$
\begin{aligned}
& \frac{1}{\omega_{\mathrm{R}}} \frac{\mathrm{d} e}{\mathrm{~d} t}=n(1+e)-\varepsilon^{\prime \prime}\left(1+2 \omega_{\mathrm{R}} \tau_{\mathrm{p}} n\right)(1+e)^{3}+\gamma^{\prime}[1+e(t-\tau)] \cos (\Delta \varphi) \\
& \frac{1}{\omega_{\mathrm{R}}} \frac{\mathrm{d} \varphi}{\mathrm{d} t}=\frac{\omega_{0}}{\omega_{\mathrm{R}}}+\alpha n-\alpha \varepsilon^{\prime \prime}\left(1+2 \omega_{\mathrm{R}} \tau_{\mathrm{p}} n\right)(1+e)^{2}-\gamma^{\prime} \frac{1+e(t-\tau)}{1+e(t)} \sin (\Delta \varphi) \\
& \frac{1}{\omega_{\mathrm{R}}} \frac{\mathrm{d} n}{\mathrm{~d} t}=-\xi \omega_{\mathrm{R}} \tau_{\mathrm{p}} n-e-2 \omega_{\mathrm{R}} \tau_{\mathrm{p}} n e-\frac{1+2 \omega_{\mathrm{R}} \tau_{\mathrm{p}} n}{2} e^{2}+\frac{\varepsilon^{\prime} \Delta J}{2}\left(1+2 \omega_{\mathrm{R}} \tau_{\mathrm{p}} n\right)(1+e)^{4}
\end{aligned}
$$

where $\gamma^{\prime}=\gamma / \omega_{\mathrm{R}}, \xi=(1+2 \Delta J) / 2 \Delta J, \varepsilon^{\prime \prime}=\varepsilon^{\prime} \Delta J /\left(2 \omega_{\mathrm{R}} \tau_{\mathrm{p}}\right)$ and $\Delta \varphi=\varphi(t)-\varphi(t-\tau)$. The original variables $E(t)$ and $N(t)$ are related to the new variables $e(t)$ and $n(t)$ by

$E(t)=\sqrt{\tau_{\mathrm{p}}\left(J-J_{\mathrm{th}}\right)}[1+e(t)]=E_{\mathrm{sol}}[1+e(t)] \quad N(t)=N_{\mathrm{th}}+\frac{2 \omega_{\mathrm{R}}}{G_{N}} n(t)$

where $\left(E_{\mathrm{sol}}, N_{\mathrm{th}}\right)$ is the steady state of the solitary laser neglecting nonlinear gain effects.

The temporal evolution of the laser variables (shown in figure 5 for the limit cycle developed from the ECM $i=3$ ) is quasi-sinusoidal with an angular frequency $\Omega \approx \omega_{\mathrm{R}}$. The phase difference between $e(t)$ and $n(t)$ is close to $\pi / 2$, while the phase differences between $n(t)$ and $\omega(t)$, and between $e(t)$ and $\Delta \phi(t)$, are small. Since we consider moderate feedback levels (such that the oscillations in the relaxation-oscillation stage are nearly harmonic), we restrict ourselves to trying to find solutions of the form

$$
\begin{aligned}
& e(t)=\bar{e}+e \sin \left(\omega_{\mathrm{R}} t\right) \\
& n(t)=\bar{n}+n\left[\cos \left(\omega_{\mathrm{R}} t\right)-\xi \omega_{\mathrm{R}} \tau_{\mathrm{p}} \sin \left(\omega_{\mathrm{R}} t\right)\right] \\
& \dot{\varphi}=\bar{\omega}+\omega \cos \left(\omega_{\mathrm{R}} t\right)+\omega_{2} \sin \left(\omega_{\mathrm{R}} t\right) \\
& \Delta \varphi(t)=\overline{\Delta \phi}_{i}+\omega_{0} \tau+\frac{2 \omega}{\omega_{\mathrm{R}}} \sin \left(\omega_{\mathrm{R}} t\right)-\frac{2 \omega_{2}}{\omega_{\mathrm{R}}} \cos \left(\omega_{\mathrm{R}} t\right) .
\end{aligned}
$$

$\bar{e}, \bar{n}, \bar{\omega}$ and $\overline{\Delta \phi} \phi_{i}+\omega_{0} \tau$ are the mean values of the variables in an oscillation period. If the ECM attractor is a torus, they are time dependent, but we are describing the relaxationoscillation stage where their time evolution is slow compared with the oscillation period, and they can be considered nearly constant. 
Table 2. Values of $\bar{e}, \bar{n}, \bar{\omega}, \overline{\Delta \phi}{ }_{i}, e, n, \omega$ and $\omega_{2}$ used in figures 5 and 6.

\begin{tabular}{rcclrllll}
\hline$i$ & $\bar{e}$ & $\bar{n}$ & $\bar{\omega}-\omega_{0}$ & \multicolumn{1}{c}{$\overline{\Delta \phi_{i}}$} & $e=n$ & $2 \omega / \omega_{\mathrm{R}}$ & $\omega_{2} / \omega_{\mathrm{R}}$ & $\bmod \left(\overline{\Delta \phi_{i}}+\omega_{0} \tau\right)$ \\
\hline-1 & -0.01 & 0.055 & 1.46 & -7.71 & 0.181 & 1.593 & -0.145 & 4.573 \\
1 & -0.01 & 0.055 & 1.46 & 4.85 & 0.183 & 1.610 & -0.147 & 4.567 \\
3 & -0.01 & 0.055 & 1.46 & 17.42 & 0.182 & 1.602 & -0.146 & 4.570 \\
6 & -0.01 & 0.055 & 1.47 & 36.27 & 0.179 & 1.575 & -0.144 & 4.571 \\
\hline
\end{tabular}
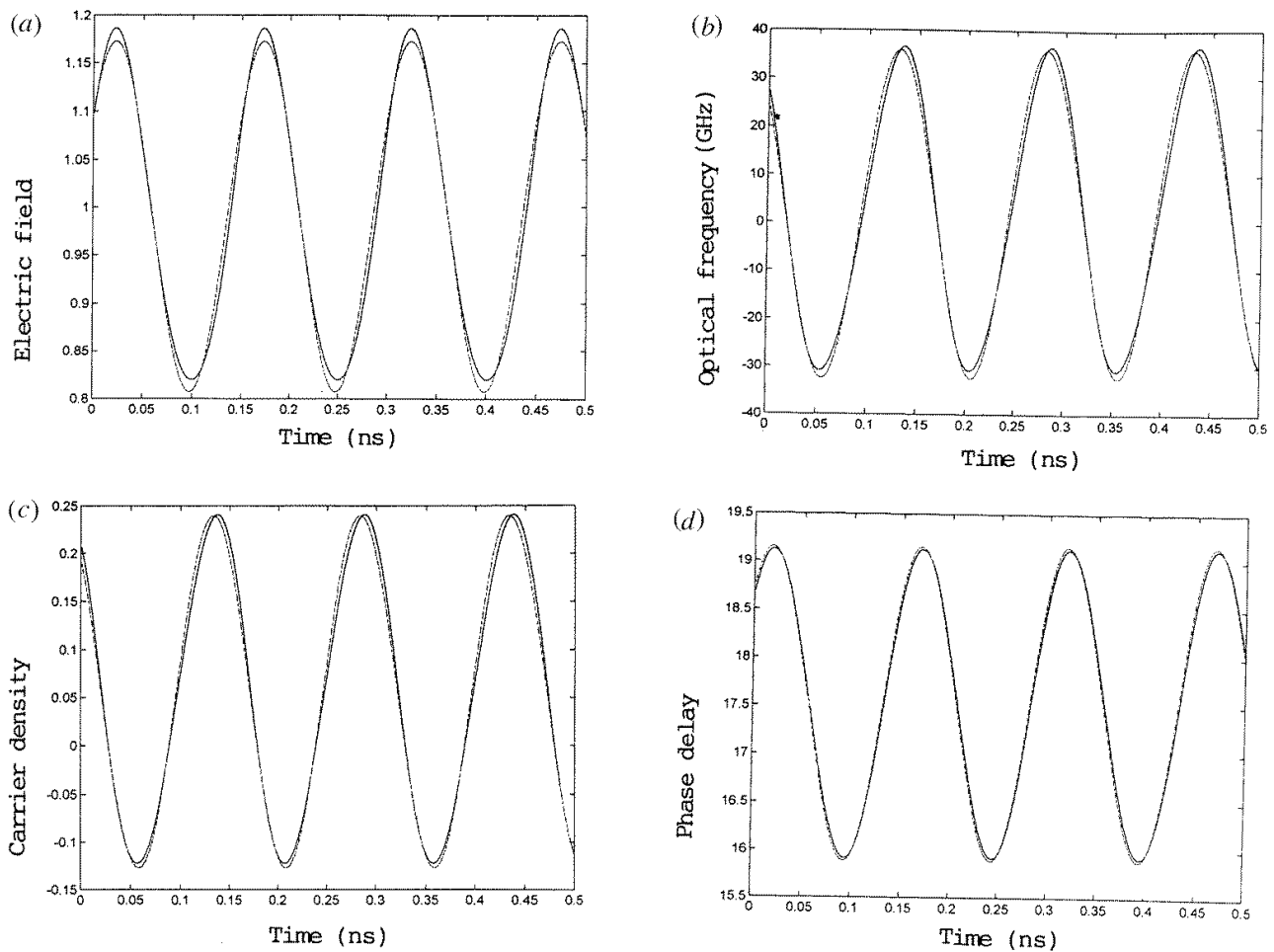

Figure 5. Temporal evolution of the laser variables $(a) 1+e(t),(b) \omega(t)=\mathrm{d} \phi / \mathrm{d} t,(c) n(t)$ and (d) $\Delta \phi(t)$ for the limit cycle of figure 4(c) (full curve). The dotted curve represents equation (21) with the values of $\bar{e}, \bar{n}, \bar{\omega}, \overline{\Delta \phi_{i}}, e, n, \omega$ and $\omega_{2}$ given in table 2 for mode $i=3$.

Although we could have included as additional variables the phases of the oscillations of $e, n$ and $\dot{\varphi}$, we prefer to keep the analysis relatively simple and easy to follow rather than precise. We choose the phases such that the phase difference between the carrier and the electric field oscillations is the one derived by Ritter and Haug [16] in the vicinity of the Hopf bifurcation $\left(2 \Gamma_{N} / \omega_{\mathrm{R}}\right.$ in equation (2.11) of [16] corresponds to $\xi \omega_{\mathrm{R}} \tau_{\mathrm{p}}=\left(1 / \tau_{\mathrm{s}}+G_{N} E_{\mathrm{sol}}^{2}\right) / \omega_{\mathrm{R}}$ in our paper). This approximation is rather good for moderate amplitudes, as can be seen in figure 5, where the dotted curve represents equation (21) with the values of $\bar{e}, \bar{n}, \bar{\omega}, \overline{\Delta \phi_{i}}, e, n, \omega$ and $\omega_{2}$ given in table 2 for the ECM $i=3$.

First, we treat the rate equation for $n$. Our numerical simulations have shown that even far from the Hopf bifurcation $\bar{e} \approx \mathrm{O}(-2), \bar{n} \approx \mathrm{O}(-2), e, n \approx \mathrm{O}(-1)$. Since $\omega_{\mathrm{R}} \tau_{\mathrm{p}} \approx \mathrm{O}(-2)$ and $\varepsilon^{\prime} \Delta J \approx \mathrm{O}(-3)$ we neglect terms in $\omega_{\mathrm{R}} \tau_{\mathrm{p}}$ ne but keep terms in $e^{2}$ and equation (19) 
becomes

$$
\frac{1}{\omega_{\mathrm{R}}} \frac{\mathrm{d} n}{\mathrm{~d} t}=-\xi \omega_{\mathrm{R}} \tau_{\mathrm{p}} n-e-\frac{1}{2} e^{2}+\frac{\varepsilon^{\prime} \Delta J}{2} .
$$

We approximate.

$$
e^{2}(t) \approx \frac{1}{2} e^{2}+2 \bar{e} e \sin \left(\omega_{\mathrm{R}} t\right) .
$$

Inserting (21) and (23) into (22) and equating coefficients we obtain

$$
\begin{aligned}
& \xi \omega_{\mathrm{R}} \tau_{\mathrm{p}} \bar{n}+\bar{e}+\frac{1}{4} e^{2}=\frac{1}{2} \varepsilon^{\prime} \Delta J \\
& -\xi \omega_{\mathrm{R}} \tau_{\mathrm{p}} n=-\xi \omega_{\mathrm{R}} \tau_{\mathrm{p}} n \\
& -n+e+\bar{e} e=0 .
\end{aligned}
$$

The advantage of the choice of the phase difference between the carrier and the electric field oscillations is now evident since equation (25) is trivial. We have assumed that $\xi$ is not too large and neglected terms in $\xi^{2} \omega_{\mathrm{R}}^{2} \tau_{\mathrm{p}}^{2}$ (this approximation is valid when the laser operates far from the solitary laser threshold).

Next, we consider the rate equations for $e$ and $\varphi$. Taking into account that $\varepsilon^{\prime \prime} \approx \mathrm{O}(-2)$ and $\gamma^{\prime} \approx \mathrm{O}(-2)$, we approximated equations (17) and (18) as

$$
\begin{aligned}
& \frac{1}{\omega_{\mathrm{R}}} \frac{\mathrm{d} e}{\mathrm{~d} t}-n(1+e)+\varepsilon^{\prime \prime}(1+3 e)=\gamma^{\prime}[1+e(t-\tau)] \cos (\Delta \varphi) \\
& \frac{1}{\omega_{\mathrm{R}}} \frac{\mathrm{d} \varphi}{\mathrm{d} t}-\frac{\omega_{0}}{\omega_{\mathrm{R}}}-\alpha n+\alpha \varepsilon^{\prime \prime}(1+2 e)=-\gamma^{\prime}[1+e(t-\tau)-e(t)] \sin (\Delta \varphi) . \\
& \text { If } \omega_{\mathrm{R}} \tau=(2 m+1) \tau,
\end{aligned}
$$

$$
e(t-\tau)=\bar{e}-e \sin \left(\omega_{\mathrm{R}} t\right) .
$$

Expanding the feedback terms in equations (27) and (28) in Bessel functions [20] gives

$$
\begin{aligned}
& {[1+e(t-\tau)] \cos (\Delta \varphi) \approx\left[1-e \sin \left(\omega_{\mathrm{R}} t\right)\right]\left[x J_{0}\left(\frac{2 \omega}{\omega_{\mathrm{R}}}\right)-2 y J_{1}\left(\frac{2 \omega}{\omega_{\mathrm{R}}}\right) \sin \left(\omega_{\mathrm{R}} t\right)\right]} \\
& \approx\left[x J_{0}\left(\frac{2 \omega}{\omega_{\mathrm{R}}}\right)+e y J_{1}\left(\frac{2 \omega}{\omega_{\mathrm{R}}}\right)\right]+\sin \left(\omega_{\mathrm{R}} t\right)\left[-2 y J_{1}\left(\frac{2 \omega}{\omega_{\mathrm{R}}}\right)-e x J_{0}\left(\frac{2 \omega}{\omega_{\mathrm{R}}}\right)\right] \\
& {[1+e(t-\tau)-e(t)] \sin (\Delta \varphi) \approx\left[1-2 e \sin \left(\omega_{\mathrm{R}} t\right)\right]\left[y J_{0}\left(\frac{2 \omega}{\omega_{\mathrm{R}}}\right)+2 x J_{1}\left(\frac{2 \omega}{\omega_{\mathrm{R}}}\right) \sin \left(\omega_{\mathrm{R}} t\right)\right]} \\
& \approx\left[y J_{0}\left(\frac{2 \omega}{\omega_{\mathrm{R}}}\right)-2 e x J_{1}\left(\frac{2 \omega}{\omega_{\mathrm{R}}}\right)\right]+\left[2 x J_{1}\left(\frac{2 \omega}{\omega_{\mathrm{R}}}\right)-2 e y J_{0}\left(\frac{2 \omega}{\omega_{\mathrm{R}}}\right)\right] \sin \left(\omega_{\mathrm{R}} t\right)
\end{aligned}
$$

where

$$
x=\cos \left(\overline{\Delta \phi}_{i}+\omega_{0} \tau\right) \quad y=\sin \left(\overline{\Delta \phi}_{i}+\omega_{0} \tau\right)
$$

We approximate

$$
n(t) e(t) \approx n \bar{e} \cos \left(\omega_{\mathrm{R}} t\right)+\bar{n} e \sin \left(\omega_{\mathrm{R}} t\right) .
$$

Inserting equations (21), (30), (31) and (33) into (27) and (28) and equating coefficients yields

$$
\begin{aligned}
& -\bar{n}+\varepsilon^{\prime \prime}=\gamma^{\prime}\left[x J_{0}\left(\frac{2 \omega}{\omega_{\mathrm{R}}}\right)+e y J_{1}\left(\frac{2 \omega}{\omega_{\mathrm{R}}}\right)\right] \\
& e-n-n \bar{e}=0
\end{aligned}
$$




$$
\begin{aligned}
& \xi \omega_{\mathrm{R}} \tau_{\mathrm{p}} n-\bar{n} e+3 \varepsilon^{\prime \prime} e=\gamma^{\prime}\left[-2 y J_{1}\left(\frac{2 \omega}{\omega_{\mathrm{R}}}\right)-e x J_{0}\left(\frac{2 \omega}{\omega_{\mathrm{R}}}\right)\right] \\
& \frac{\bar{\omega}}{\omega_{\mathrm{R}}}-\frac{\omega_{0}}{\omega_{\mathrm{R}}}-\alpha \bar{n}+\alpha \varepsilon^{\prime \prime}=-\gamma^{\prime}\left[y J_{0}\left(\frac{2 \omega}{\omega_{\mathrm{R}}}\right)-2 e x J_{1}\left(\frac{2 \omega}{\omega_{\mathrm{R}}}\right)\right] \\
& \frac{\omega}{\omega_{\mathrm{R}}}=\alpha n \\
& \frac{\omega_{2}}{\omega_{\mathrm{R}}}+\alpha \xi \omega_{\mathrm{R}} \tau_{\mathrm{p}} n+2 \alpha \varepsilon^{\prime \prime} e=-\gamma^{\prime}\left[2 x J_{1}\left(\frac{2 \omega}{\omega_{\mathrm{R}}}\right)-2 e y J_{0}\left(\frac{2 \omega}{\omega_{\mathrm{R}}}\right)\right] .
\end{aligned}
$$

The first-order approximation of equations (26) and (35) gives

$$
e \approx n \text {. }
$$

Inserting equations (38) and (40) into equations (34) and (36) yields

$$
\left(\xi \omega_{\mathrm{R}} \tau_{\mathrm{p}}+2 \varepsilon^{\prime \prime}\right) \frac{\omega}{\alpha \omega_{\mathrm{R}}}=-2 \gamma^{\prime} J_{1}\left(\frac{2 \omega}{\omega_{\mathrm{R}}}\right) \sin \left(\overline{\Delta \phi_{i}}+\omega_{0} \tau\right) .
$$

Inserting equations (38) and (40) into equations (34) and (37) yields

$$
\frac{\bar{\omega}}{\omega_{\mathrm{R}}}-\frac{\omega_{0}}{\omega_{\mathrm{R}}}=-\gamma^{\prime}\left\{\alpha\left[x J_{0}\left(\frac{2 \omega}{\omega_{\mathrm{R}}}\right)+2 e y J_{1}\left(\frac{2 \omega}{\omega_{\mathrm{R}}}\right)\right]+\left[y J_{0}\left(\frac{2 \omega}{\omega_{\mathrm{R}}}\right)-2 e x J_{1}\left(\frac{2 \omega}{\omega_{\mathrm{R}}}\right)\right]\right\}
$$

which in the limit $e=n=\omega=0$ reduces to equation (4). Similar relations, but derived near the Hopf bifurcation, were found in [16] $\left(a_{I} / \bar{I}, a_{N}, a_{\phi}\right.$ of [16] correspond to $2 e, 2 \omega_{\mathrm{R}} n / G_{N}, 2 \omega / \omega_{\mathrm{R}}$ in our paper; equations (2.12), (2.21) and (2.34) of [16] correspond
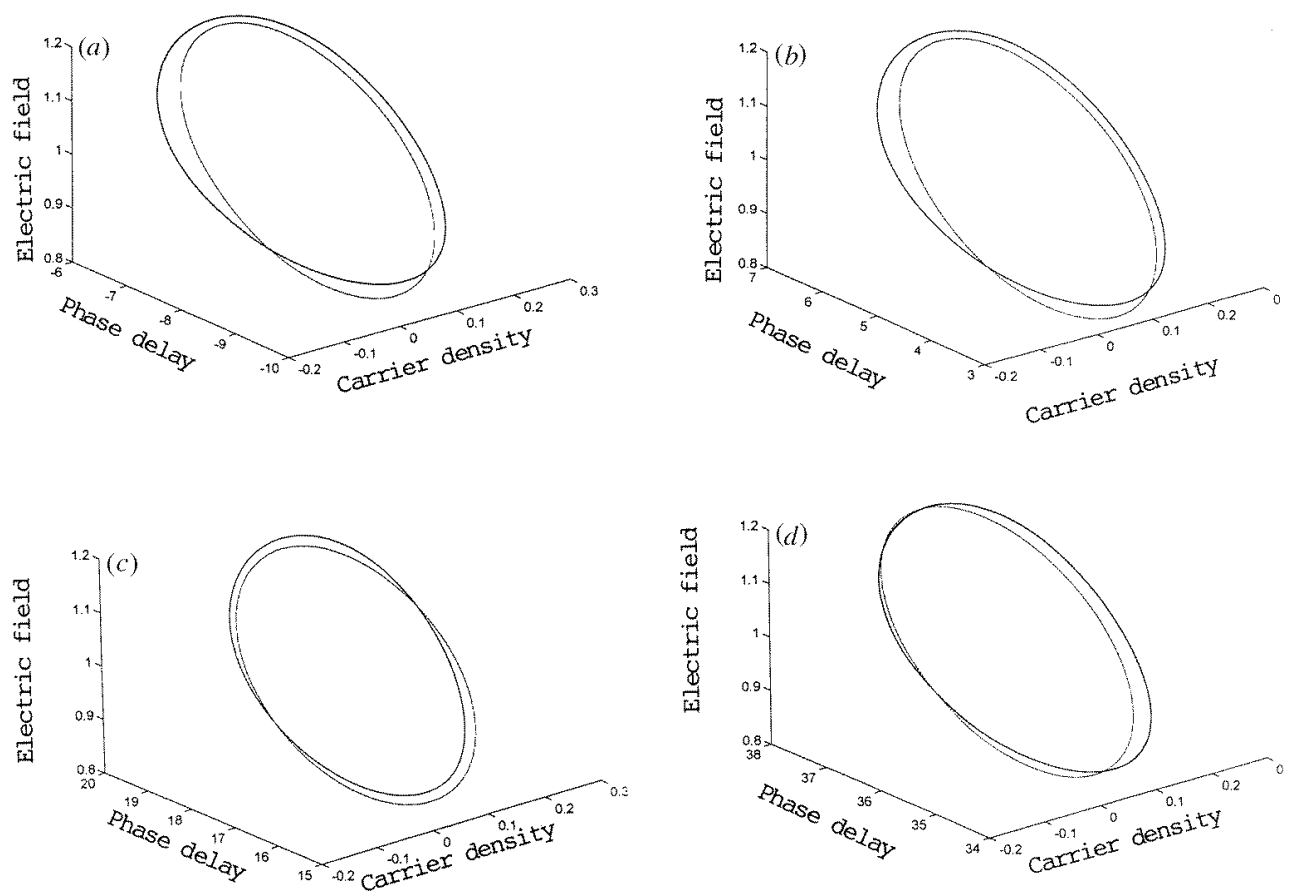

Figure 6. Relaxation-oscillation stage, for the same ECM attractors and parameters as figure 4. The full curve represents the numerical simulation, and the broken curve equation (21) with the values of $\bar{e}, \bar{n}, \bar{\omega}, \overline{\Delta \phi}{ }_{i}, e, n, \omega$ and $\omega_{2}$ given in table 2 . 
to equations (40), (42) and (38) of our paper). In the limit of a large value of $\alpha$, from equation (41) the value of $2 \omega / \omega_{\mathrm{R}}$ is a root of the Bessel function $J_{1}(x)$, in agreement with the results of [17].

We arrive at seven equations (equations (24), (34), (38)-(42)) which have eight unknowns $\left(\bar{e}, \bar{n}, \bar{\omega}, \overline{\Delta \phi}_{i}, e, n, \omega\right.$ and $\left.\omega_{2}\right)$. To solve this system of equations, we proceed as follows: from the numerical simulation we estimate the value of $n$. Then, from equations (38) and (40) we calculate the values of $e$ and $\omega$; from equation (41) we find the value of $\overline{\Delta \phi_{i}}$, and from equation (34), we then find the value of $\bar{n}$. From equation (24) we calculate the value of $\bar{e}$; from equation (39), we find the value of $\omega_{2}$, and from equation (42), we finally determine the value of $\bar{\omega}$.

In figure 6 we compare our results with numerical simulations of the original rate equations. The full curve represents the trajectory in the relaxation-oscillation stage from the numerical simulation (for the same ECM attractors shown in figure 4), while the broken curve represents equation (21) with the values of $\bar{e}, \bar{n}, \bar{\omega}, \overline{\Delta \phi}{ }_{i}, e, n, \omega$ and $\omega_{2}$ given in table 2. There is relatively good agreement, even for those ECM attractors in which the feedback level is much larger than the critical feedback where the excitation of relaxation oscillations occurs. The values of $\bar{e}, \bar{n}, \bar{\omega}, e, n, \omega$ and $\omega_{2}$ are almost the same for all the ECM attractors.

\section{Summary and conclusions}

In summary, we have studied the stability and modulation properties of a single-longitudinalmode semiconductor laser biased well above threshold and subjected to optical feedback from a distant reflector. We have found that for moderately strong feedback, there are some pairs of external cavity modes that are both unstable when they are created (one is an antimode, and the other is an unstable mode). Since the antimodes are usually associated with destructive interference (between the field inside the laser cavity and the delayed field returning from the external cavity), and the modes, with constructive interference, it is a surprising result that some modes are unstable when they first appear.

The unstable modes have a large positive frequency shift with respect to the operating frequency of the solitary laser, and they are located in phase space on the opposite side of the high power modes, which are stable, but which have very narrow basins of attraction.

The fact that pairs of modes and antimodes might appear both initially unstable, seems not to have been reported previously. They might have applications in the use of laser diodes with optical injection which are also under the influence of optical feedback. If there is a large positive frequency shift of the injected field from $\omega_{0}$, locking might not be achieved because there might be no attracting points in that frequency region. This phenomenon may also be useful in chaos-preventing techniques in which the key point is choosing an initially stable external cavity mode (see, for example, [21]).

We have also studied in detail the modulation characteristics when the laser operates on ECM attractors which have mean optical frequencies near $\omega_{0}$. Moderately strong optical feedback induces a modulation that is either periodic or quasiperiodic, depending on the frequency of the ECM from which these attractors originate. For quasiperiodic modulation, there is a stage which is almost periodic. We studied analytically this nearly periodic stage, and found that a first-order Fourier expansion of the laser variables is enough to describe the dynamics accurately. The amplitude of the oscillations of the electric field, carrier density 
and optical frequency are related by

$$
\frac{E(t)}{E_{\mathrm{sol}}}-1=\frac{G_{N} N_{\mathrm{th}}}{2 \omega_{\mathrm{R}}}\left[\frac{N(t)}{N_{\mathrm{th}}}-1\right]=\frac{\omega(t)}{\alpha \omega_{\mathrm{R}}}
$$

which is independent of the particular ECM attractor. This suggest that the individual ECM attractors may have their full identity masked from identification by measurements of their intensity fluctuations. Our results were derived assuming $\omega_{\mathrm{R}} \tau=(2 m+1) \tau$, but numerical simulation reveal that these results hold even if this condition is not fulfilled (as long as the delay time is long, with respect to the oscillation period). Our results are valid when the laser is operated well above threshold, since we assumed a small value of $\xi \omega_{\mathrm{R}} \tau_{\mathrm{p}}$.

\section{Acknowledgments}

This work was supported by the Consejo Nacional de Investigaciones Científicas y Técnicas (CONICYT), the Comisión Sectorial de Investigación Científica (CSIC), and the Proyecto de Desarrollo de Ciencias Básicas (PEDECIBA).

\section{References}

[1] Agrawal G P and Dutta N K 1986 Long-Wavelength Semiconductor Lasers (New York: Van Nostrand Reinhold)

[2] Lenstra D, Verbeck B H and den Boef A J 1985 Coherence collapse in single-mode semiconductor lasers due to optical feedback IEEE J. Quantum Electron. QE-21 674-9

[3] Mørk J, Tromborg B and Mark J 1992 Chaos in semiconductor lasers with optical feedback: theory and experiment IEEE J. Quantum Electron. QE-28 93-107

[4] Lang R and Kobayashi K 1980 External optical feedback effects on semiconductor injection laser properties IEEE J. Quantum Electron. QE-16 347-55

[5] Tromborg B, Osmundsen J H and Olesen H 1984 Stability analysis for a semiconductor laser in an external cavity IEEE J. Quantum Electron. QE-20 1023-32

[6] Ritter A and Haug H 1993 Theory of laser diodes with weak optical feedback. I. Small-signal analysis and side-mode spectra J. Opt. Soc. Am. B 10 130-44

[7] Erneux T, van Tartwijk G, Lentra D and Levine A M 1995 Determining Lang and Kobayashi Hopf bifurcation points Proc. SPIE 2399170

[8] Erneux T, Alsing P M, Kovanis V and Gavrielides A 1996 Lang and Kobayashi phase equation and its validity for low pump Proc. SPIE 2693 701-9

[9] Lythe G, Erneux T, Gavrielides A and Kovanis V 1997 Low pump limit of the bifurcation to periodic intensities in a semiconductor laser subject to external optical feedback Phys. Rev. A 55 4443-8

[10] Levine A M, van Tartwijk G H M, Lenstra D and Erneux T 1995 Diode lasers with optical feedback: stability of the maximum gain mode Phys. Rev. A 52 R3436-9

[11] Masoller C and Abraham N B 1998 Stability and dynamical properties of the coexisting attractors of an external cavity semiconductor laser Phys. Rev. A 57 1313-22

[12] Mørk J and B Tromborg 1990 The mechanism of mode selection for an external cavity laser IEEE Photonics Technol. Lett. 2 21-3

[13] Hernandez-Garcia E, Abraham N B, San Miguel M and De Pasquale F 1992 Frequency selection and transient dynamics in single-mode lasers with optical feedback J. Appl. Phys. 72 1225-36

[14] Mørk J, Mark J and B Tromborg 1990 Route to chaos and competition between relaxation oscillations for a semiconductor laser with optical feedback Phys. Rev. Lett. 65 1999-2002

[15] Ritter A and Haug H 1993 Theory of the bistable limit cycle behavior of laser diodes induced by weak optical feedback IEEE J. Quantum Electron. QE-29 1064-70

[16] Ritter A and Haug H 1993 Theory of laser diodes with weak optical feedback. II. Limit-cycle behavior, quasi-periodicity, frequency locking, and route to chaos J. Opt. Soc. Am. B 10145

[17] Alsing P M, Kovanis V, Gavrielides A and Erneux T 1996 Lang and Kobayashi phase equation Phys. Rev. A 534429

[18] De Jagher R C, van der Graaf W A and Lenstra D 1996 Relaxation-oscillation phenomena in an injectionlocked semiconductor laser Quantum Semiclass. Opt. 8 805-22 
[19] van der Graaf W A, Levine A M and Lenstra D 1997 Diode lasers locked to noisy injection IEEE J. Quantum Electron. QE-33 434-42

[20] Abramowitz M and Stegun I A 1972 Handbook of Mathematical Functions (New York: Dover)

[21] Wieland J, Mirasso C R and Lenstra D 1997 Prevention of coherence collapse in diode lasers by dynamic targeting Opt. Lett. 22 469-71 